\title{
Estimating soil thermal diffusivity with interference analyses
}

\author{
Thomas Mimouni $\cdot$ Lingxi Lei $\cdot$ Lyesse Laloui
}

Received: 24 September 2013/Accepted: 4 April 2014/Published online: 25 May 2014

(C) Springer-Verlag Berlin Heidelberg 2014

\begin{abstract}
The development of ground source heat pumps has facilitated the use of geothermal power at shallow depths. Initially, ground heat exchangers were buried in trenches or boreholes, but recent investigations and increasing congestion of landscapes have suggested the use of foundation structures as heat exchangers with the ground. Foundations are shorter than conventional borehole heat exchangers and are closer to each other to ensure structural support. The thermal inertia of the ground wherein seasonal heat storage is achieved, therefore, becomes an important parameter for the design of such structures. Although thermal response tests have been developed to estimate bulk thermal conductivity on the scale of geothermal boreholes, only laboratory or shallow in situ test methods have been developed to estimate the thermal diffusivity of the ground. This paper investigates the potential for using a periodic pumping test procedure to measure the thermal diffusivity of soils in a scaled model of a geothermal borehole. The thermal diffusivity estimates obtained are in agreement with values reported in the literature and illustrate the potential of the proposed method.
\end{abstract}

Keywords Ground heat exchanger - In situ test . Interference analysis - Periodic pumping test - Phase shift · Signal attenuation - Thermal diffusion - Thermal diffusivity

T. Mimouni $(\bowtie) \cdot$ L. Lei $\cdot$ L. Laloui

Laboratory of Soil Mechanics, Swiss Federal Institute of Technology Lausanne, Station 18, 1015 Lausanne, Switzerland e-mail: thomas.mimouni@epfl.ch

L. Laloui

King Abdulaziz University, Jeddah, Saudi Arabia

\author{
List of symbols \\ $\lambda$ Bulk soil thermal conductivity $(\mathrm{W} / \mathrm{m} / \mathrm{K})$ \\ $\rho \quad$ Bulk soil density $\left(\mathrm{kg} / \mathrm{m}^{3}\right)$ \\ $c \quad$ Bulk soil heat capacity $(\mathrm{J} / \mathrm{kg} / \mathrm{K})$ \\ $D_{\mathrm{T}}=\lambda / \rho c \quad$ Bulk soil thermal diffusivity $\left(\mathrm{m}^{2} / \mathrm{s}\right)$ \\ $D_{\varphi} \quad$ Thermal diffusivity based on phase shift \\ analyses $\left(\mathrm{m}^{2} / \mathrm{s}\right)$ \\ $D_{\delta} \quad$ Thermal diffusivity based on amplitude \\ attenuation $\left(\mathrm{m}^{2} / \mathrm{s}\right)$ \\ $T \quad$ Soil absolute temperature $\left({ }^{\circ} \mathrm{C}\right.$ or $\left.\mathrm{K}\right)$ \\ $T_{0} \quad$ Ambient laboratory temperature, also mean \\ soil temperature $\left({ }^{\circ} \mathrm{C}\right.$ or $\left.\mathrm{K}\right)$ \\ $\theta=T-T_{0} \quad$ Soil temperature anomaly $\left({ }^{\circ} \mathrm{C}\right.$ or $\left.\mathrm{K}\right)$ \\ $\theta_{0} \quad$ Amplitude of soil temperature anomaly at \\ the heat source shaft $\left({ }^{\circ} \mathrm{C}\right.$ or $\left.\mathrm{K}\right)$ \\ $\hat{T}, \hat{\theta} \quad$ Complex representations of soil temperature \\ and soil temperature anomaly \\ $r, r_{i}, R \quad$ Radial coordinate, radius of the heat source \\ and radius of the soil sample (m) \\ $t \quad$ Time (s) \\ $\tau \quad$ Period of the heat source signal (s) \\ $\omega=2 \pi / \tau \quad$ Pulsation of the heat source signal ( $\mathrm{rad} / \mathrm{s})$ \\ $\delta_{\mathrm{TT}} \quad$ Amplitude attenuation \\ $\varphi_{\mathrm{TT}} \quad$ Phase shift
}

Mathematics

$I_{n}, K_{n} \quad$ Modified Bessel functions of $n$th order

$i \quad$ Imaginary unit

\section{Introduction}

Shallow geothermal energy represents a substantial reservoir of heat [3], the high efficiency use of which has been made possible by the development of ground source heat pumps (GSHPs). Two types of GSHP systems can be 
distinguished on the basis of the type of ground heat exchanger (GHE) that is used [19]: open-loop and closedloop systems. In open-loop systems, water from an aquifer is pumped, processed and injected back downstream. This type of system requires particular conditions (the presence of an aquifer with a favourable permeability) and significant maintenance (to prevent clogging and corrosion of the wells). Closed-loop systems use embedded absorber pipes that are in contact with the ground, and these pipes circulate a heat-carrier fluid, allowing heat exchanges with the heat pump. Therefore, closed-loop systems are widely installed in various types of stratigraphy, the limiting factor being the soil drillability. This paper focuses on closedloop systems.

The proper design of closed-loop GSHPs requires a good knowledge of the in situ soil conditions, which can be classified in two main categories. The first one corresponds to soils in which a significant natural underground water flow exists. In this case, the soil conditions are not favourable for seasonal heat storage, as the injected heat would be advected away from the GHEs, but are favourable for providing a heat source with a rather stable temperature all year long. The second category corresponds to soils in which no significant underground water flow is observed. In this case, seasonal heat storage is recommended to ensure the sustainability of the heat source. The present paper focuses on the second category of soil conditions, in which no natural underground water flow is observed.

The thermal inertia of the ground is of great importance in ensuring the sustainability of the heat storage as well as the efficiency of the GSHP system. The sensitivity of GSHP systems to design and applied loads was evidenced by Garber et al. [9]. Furthermore, the use of foundation structures as heat exchangers with the ground leads to shorter and more closely spaced GHEs which makes the thermal inertia of the ground even more important.

Under the stated ground conditions, the main heat transport process is heat conduction [10], which is characterised by the thermal conductivity, thermal diffusivity and/or heat capacity of the ground. Therefore, great efforts have been applied to determine these characteristics. Farouki [7] separates the methods to estimate the thermal properties of soils into two categories: those based on steady-state analyses and those based on transient analyses.

The steady-state analysis methods are mainly used to determine the thermal conductivity of soils once a heat flux is established either through a sample (using a guarded hot plate [2] or a cylindrical arrangement [13]) or in situ (using the sphere method [16] or a heat meter [20]).

Transient methods provide information about the thermal inertia of the soil, i.e., its thermal diffusivity. Forbes et al. [8] analysed the propagation of temperature waves in soil using transient one-dimensional (vertical) heat transport analyses. Hoekstra et al. [12] estimated the thermal diffusivity of cylindrical soil samples by applying sinusoidal temperature signals to the sides of the samples and measuring the temperature variations at the centres. Shannon and Wells [21] used a thermal shock procedure (i.e. a sudden change in temperature that is maintained) to estimate the thermal diffusivity of a cylindrical sample.

However, these different methods do not provide estimates at an operational scale for GHEs that reach depths between 10 and $100 \mathrm{~m}$. Samples barely represent real in situ conditions, and in situ techniques such as the dualprobe pulse method [5] (that is also used for laboratory testing) and time series analyses of one-dimensional vertical heat conduction data [11] only permit investigation of the thermal diffusivity of soils within the first few metres below the surface. Indeed, the former technique is limited by the size of the probes [5], while the latter is limited by the relatively shallow depths, not exceeding 3-5 m, within which the temperature varies annually [22].

Thermal response tests (TRTs) have been developed based on the infinite line source model [6] to estimate the bulk thermal conductivity of a soil mass in which a geothermal borehole has been installed [15]. However, the inertia of soils has not yet been investigated on such a scale, even though the bulk thermal diffusivity of soils is becoming more important as shorter and more concentrated GHEs (i.e. energy geostructures [14]) are increasingly being used.

Therefore, this paper investigates the potential of using a periodic pumping test that was originally designed to determine the in situ hydraulic properties of soils [18] to estimate the bulk thermal diffusivity of a soil mass on a borehole scale. This estimation was accomplished using an experimental scaled model of a geothermal borehole. The mathematical basis of the method, including the derivation of the equations involved and the graphical inversion used to retrieve thermal diffusivity values from the experimental data based on interference analyses, is presented in Sect. 2. The scaled model used in the present study is described in Sect. 3, as well as the data processing method. Finally, the results obtained based on source-thermocouple and thermocouple-thermocouple interference analyses are presented in Sect. 4. Section 5 discusses the applicability of this method on a real scale, based on the scaled model results.

\section{Testing methodology}

Determining the physical (hydraulic or thermal) properties of soils under a forced periodic regime is achieved by comparing an input signal to a recorded signal at a given 
distance from the source. Comparisons are achieved by means of phase shift and attenuation analyses. The phase shift represents a certain delay between the source and the recorded signal and is related to the inertia of the system. Attenuation is also observed between the amplitude of the source signal and the monitored response. This attenuation is characteristic of storage and/or dissipative effects between the source and the measuring point. This type of analysis is called interference analysis because comparisons are achieved between two different points [18].

\section{a. Mathematical formulation}

Heat transport within soils can be achieved through many different processes [4] but thermal diffusion is the main process occurring in soils in which water advection is negligible [10]. This process is governed by the so-called heat equation:

$\rho c \frac{\partial T}{\partial t}=\nabla(\lambda \cdot \nabla T)+Q_{\mathrm{T}}$

where $\lambda, \rho$ and $c$ are the bulk thermal conductivity, density and heat capacity of the soil, respectively, and $Q_{\mathrm{T}}$ represents any volumetric heat source or sink.

Because the present study focuses on determining the bulk thermal properties of a soil under a forced periodic regime imposed through an "injection well" [18], the geometry of the source is axisymmetric, and one may want to transpose Eq. (1) into cylindrical coordinates. Furthermore, if the soil is assumed to be isotropic from a thermal standpoint and it is assumed that no volumetric heat sources or sinks are present, the heat Eq. (1) becomes:

$\frac{\partial T}{\partial t}=D_{\mathrm{T}}\left\{\frac{\partial^{2} T}{\partial r^{2}}+\frac{1}{r} \frac{\partial T}{\partial r}\right\}$

where $r$ is the distance to the source axis and $D_{\mathrm{T}}$ is the bulk thermal diffusivity of the soil, defined as:

$D_{\mathrm{T}}=\frac{\lambda}{\rho c}$

The temperature anomaly applied to the soil at the soilGHE interface (i.e. at $r=r_{i}$ ) is a sinusoidal variation that is assumed to be homogeneous along the GHE wall. Therefore, the applied temperature anomaly can be modelled with a complex form, expressed as:

$$
\begin{aligned}
& \hat{\theta}\left(r=r_{i}, t\right)=\hat{\theta}_{i}(t)=\theta_{0} \exp (i \omega t) \\
& \theta\left(r=r_{i}, t\right)=\operatorname{Re}\left\{\hat{\theta}\left(r=r_{i}, t\right)\right\}
\end{aligned}
$$

where $\omega=2 \pi / \tau$ is the signal pulsation, $\tau$ is the period of the source signal and $\theta_{0}$ its amplitude. Inserting the following temperature anomaly equation:

$\hat{\theta}(r, t)=\hat{T}(r, t)-T_{0}$ where $T_{0}$ is the natural field temperature, into Eq. (2) yields the following equation:

$\frac{\partial \hat{\theta}}{\partial t}=D_{\mathrm{T}}\left\{\frac{\partial^{2} \hat{\theta}}{\partial r^{2}}+\frac{1}{r} \frac{\partial \hat{\theta}}{\partial r}\right\}$

Spreading the space and time variables and searching for a solution with the same pulsation as the source signal [Eq. (4)] yields the following equations:

$$
\begin{aligned}
& \hat{\theta}(r, t)=\theta_{r}(r) \exp (i \omega t) \\
& \theta(r, t)=\operatorname{Re}\{\hat{\theta}(r, t)\}
\end{aligned}
$$

Combining Eq. (7) with Eq. (6) yields the following equation:

$r^{2} \frac{\partial^{2} \theta_{r}(r)}{\partial r^{2}}+r \frac{\partial \theta_{r}(r)}{\partial r}-\left(r \sqrt{\frac{i \omega}{D_{T}}}\right)^{2} \quad \theta_{r}(r)=0$

Equation (8) is a modified Bessel equation of zeroth order whose solution is given by a linear combination of the modified Bessel functions of zeroth order $I_{0}$ and $K_{0}$ :

$\theta_{r}(r)=A \cdot I_{0}(\eta r)+B \cdot K_{0}(\eta r)$

where $\eta=\sqrt{i \omega D_{\mathrm{T}}^{-1}}$. From this general solution, two types of analyses can be developed, depending on the type of boundary conditions that are applied. Considering a fixed temperature at a given distant $R$ from the source (the Dirichlet condition) yields the following equation:

$\theta(r, t)=\frac{I_{0 R} \cdot K_{0}(\eta r)-K_{0 R} \cdot I_{0}(\eta r)}{I_{0 R} \cdot K_{0 r_{i}}-K_{0 R} \cdot I_{0 r_{i}}} \theta_{i}(t)$

where $I_{0 R}=I_{0}(\eta R), \quad I_{0 r_{i}}=I_{0}\left(\eta r_{i}\right), \quad K_{0 R}=K_{0}(\eta R) \quad$ and $K_{0 r_{i}}=K_{0}\left(\eta r_{i}\right)$ are constants. Considering an adiabatic wall at a distance $R$ from the source (the Neumann condition) yields the following equation:

$\theta(r, t)=\frac{K_{1 R} \cdot I_{0}(\eta r)+I_{1 R} \cdot K_{0}(\eta r)}{K_{1 R} \cdot I_{0 r_{i}}+I_{1 R} \cdot K_{0 r_{i}}} \theta_{i}(t)$

where $I_{1}$ and $K_{1}$ are the modified Bessel functions of the first kind and $K_{1 R}=K_{1}(\eta R)$ and $I_{1 R}=I_{1}(\eta R)$ are constants. However, in both cases, letting $R$ approach infinity (i.e. the far-field condition) yields the following equation:

$\theta(r, t)=\frac{K_{0}(\eta r)}{K_{0}\left(\eta r_{i}\right)} \theta_{i}(t)$

Based on Eq. (12), one can define an amplitude attenuation $\delta_{\mathrm{TT}}$ and a phase shift $\varphi_{\mathrm{TT}}$ between the source and a point (at $r$ ) as follows:

$$
\begin{aligned}
\delta_{\mathrm{TT}} & =\left|\frac{\theta(r, t)}{\theta_{i}(t)}\right|=\left|\frac{K_{0}(\eta r)}{K_{0}\left(\eta r_{i}\right)}\right| \\
\varphi_{\mathrm{TT}} & =\arg \left\{\frac{\theta(r, t)}{\theta_{i}(t)}\right\}=\arg \left\{\frac{K_{0}(\eta r)}{K_{0}\left(\eta r_{i}\right)}\right\}
\end{aligned}
$$



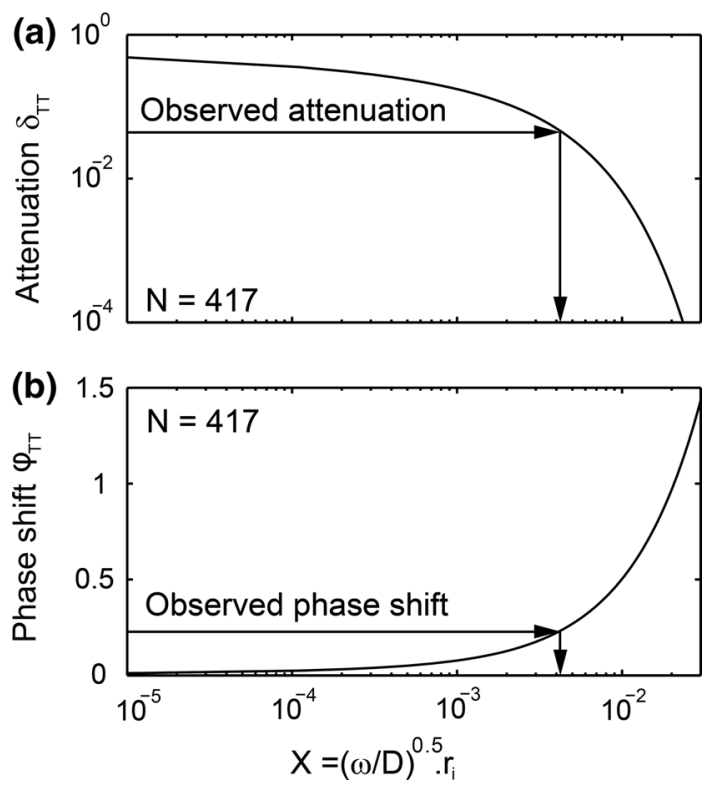

Fig. 1 Illustration of the graphical inversion of Eq. (14) with the amplitude attenuation and phase shift

\section{b. Graphical inversion of attenuation and phase shift}

In practice, the amplitude attenuation $\delta_{\mathrm{TT}}$ and phase shift $\varphi_{\mathrm{TT}}$ are experimentally determined from temperature time series recorded at the source and at a distance $r$. A graphical inversion is then used to derive the thermal diffusivity $D_{\mathrm{T}}$ because Eq. (13) is not reversible. To accomplish this inversion, Eq. (13) is rewritten as follows:

$$
\begin{aligned}
& \delta_{\mathrm{TT}}(X)=\left|\frac{K_{0}\left(N \sqrt{i}\left[\sqrt{\frac{\omega}{D_{T}}} r_{i}\right]\right)}{K_{0}\left(\sqrt{i}\left[\sqrt{\frac{\omega}{D_{T}}} r_{i}\right]\right)}\right|=\left|\frac{K_{0}(N \sqrt{i} X)}{K_{0}(\sqrt{i} X)}\right| \\
& \varphi_{\mathrm{TT}}(X)=\arg \left\{\frac{K_{0}(N \sqrt{i} X)}{K_{0}(\sqrt{i} X)}\right\}
\end{aligned}
$$

where $N=r / r_{i}$. Curves $\delta_{\mathrm{TT}}(X)$ and $\varphi_{\mathrm{TT}}(X)$ are then used to estimate values of $X$ corresponding to the observed amplitude attenuation and phase shift (Fig. 1) and consequently values of thermal diffusivity $D_{\mathrm{T}}$, using the following equation:

$X=\sqrt{\frac{\omega}{D_{T}}} r_{i} \Rightarrow D_{\mathrm{T}}=\frac{2 \pi}{\tau}\left(\frac{r_{i}}{X}\right)^{2}$

\section{Experimental set-up}

The pumping test method was investigated on a scaled model of borehole in which the soil homogeneity and the positions of the temperature sensors are much easier to control than in situ. Furthermore, the scaled model is logistically simpler, less expensive and makes it possible to have several sensors embedded in the soil around the heat source to better evaluate the potential of the method. The main features of this method are the application of a sinelike temperature signal at the heat source that mimics seasonal operations of a ground heat exchanger and the recording of the temperature anomaly in the ground at a given distance from the source. The thermal diffusivity can then be estimated by comparing the time lag and the attenuation in amplitude between the source signal and the signals measured in the ground.

a. Scaled model

A scaled model of borehole heat exchanger was developed at laboratory scale (Fig. 2). The absorber pipes consist of two collocated U-shaped loops made of copper. The equivalent diameter of the borehole is $0.01 \mathrm{~m}$. The buried length of the U-loops is $0.6 \mathrm{~m}$. These pipes are buried within a cylindrical tank whose inner diameter is $0.31 \mathrm{~m}$. The tank wall is made of steel and is $0.04 \mathrm{~m}$ thick. A top cap is used to apply a vertical load to the filling material. Two apertures (one at the base of the tank and the other in the top cap) were managed to allow water drainage resulting from the consolidation of the filling material that is installed in slightly oversaturated conditions. The aperture in the top cap also allowed connecting the scaled borehole to the heater/chiller. Holes in the tank wall, $20 \mathrm{~cm}$ above the tank bottom, were used to install the thermocouples around the scaled borehole.

The filling material is Bioley silt. This material was tested in details by Péron et al. [24]. The tank was filled layer by layer up to a level of $0.6 \mathrm{~m}$.

The heater/chiller device is a temperature controlled tank equipped with a pump delivering a flow rate of $5.4 \mathrm{~L} /$ min. The heating is provided by electrical heaters. The cooling is provided using a cryostat immersed in the tank. This cryostat had a constant cooling rate so that when the temperature of the heat carried fluid dropped below the set point, heaters were activated.

The heat carried fluid used in the absorber pipes is a mixture of water and glycol. Although no negative temperature was investigated in this study, the glycol prevented any ice to from around the cryostat which would have reduced its cooling rate.

The construction of the laboratory test was as follows:

- The porous stone was placed on top of the bottom hole of the tank

- The absorber pipes were installed and maintained vertical on top of the porous stone 

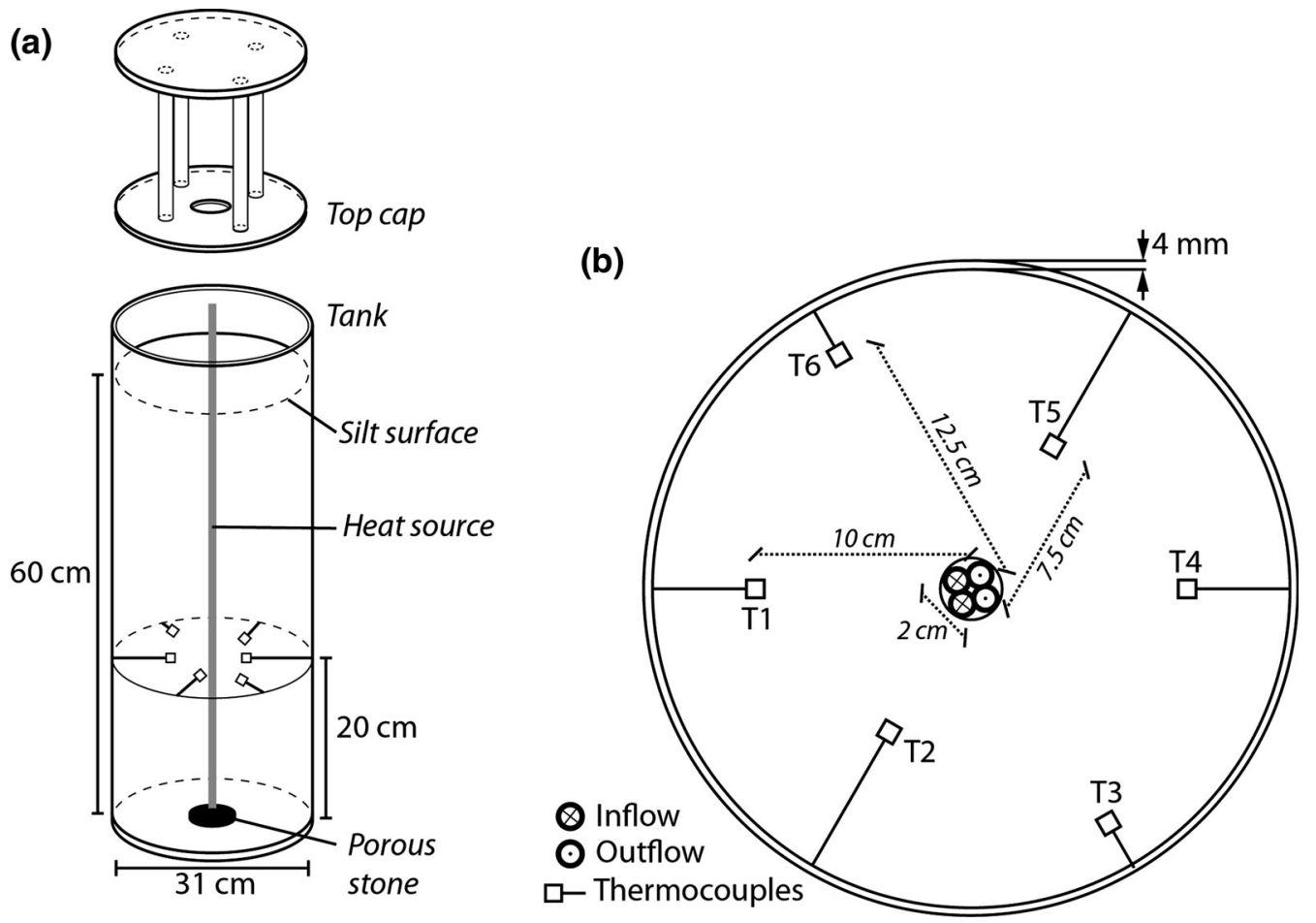

Fig. 2 a 3D schematic of the scaled model tank and $\mathbf{b}$ cross section of the set-up showing the thermocouples and absorber pipes

- The tank was filled layer by layer up to the level of $0.2 \mathrm{~m}$. Although no compaction was achieved between the installations of two layers, particular attention was given to avoid air pockets along the sidewalls and around the absorber pipes. The filling material was levelled by hand. Therefore, the thermal contact between the silt and the absorber pipes might be better than in full-scale practice.

- Thermocouples were placed on top of the silt at the desired distance from the absorber pipes.

- The tank was filled layer by layer up to $0.6 \mathrm{~m}$

- The top cap was placed on top of the tank. A toric joint was used to seal the top cap-tank contact. The top cap was then loaded with $100 \mathrm{~kg}$, and its settlements were monitored using a mechanical comparator. The scaled model was ready to use when the measured settlements did not show significant increase from 1 day to the other.

Heating and cooling cycles were produced by alternating the temperature set point of the temperature controlled tank between $\mathrm{T}_{0}+15{ }^{\circ} \mathrm{C}$ and $\mathrm{T}_{0}-15^{\circ} \mathrm{C}$, where $\mathrm{T}_{0}$ is the ambient temperature of the laboratory, maintained at approximately $23{ }^{\circ} \mathrm{C}$. The period adopted was $24 \mathrm{~h}$, so the temperature set point is changed every $12 \mathrm{~h}$. The test described here was conducted over 3.5 cycles.

Six thermocouples were deployed around the absorber pipes. Two additional thermocouples were installed right at the inlet and outlet of the absorber pipes to monitor the

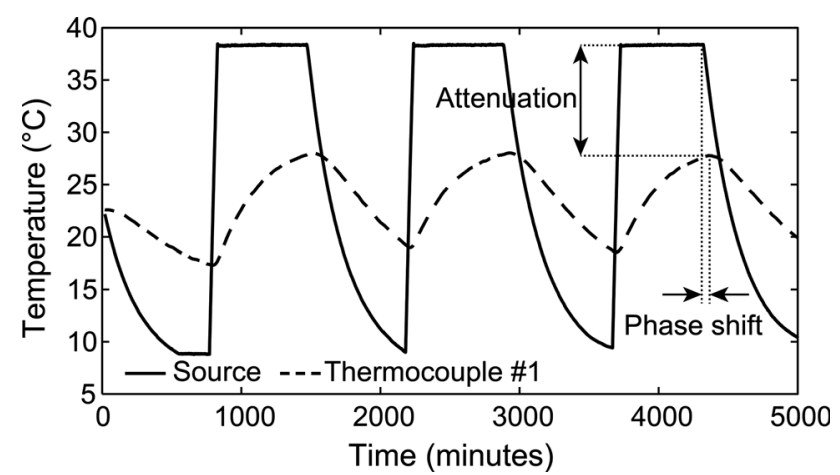

Fig. 3 Example of source-thermocouple analysis, here between the source and thermocouple $\mathrm{T} 1$

temperature of the inflow and outflow of heat-carrier fluid. This allowed getting rid of thermal losses occurring within the pipes connecting the scaled borehole to the temperature controlled tank. Because of the consolidation occurring after the installation of the thermocouples, these might have slightly moved downward, slightly increasing their distance to the absorber pipes. However, this was not quantified and therefore assumed to be negligible for these analyses. Facing thermocouples were installed at the same distances from the absorber pipes so that data for three different distances to the source axis were obtained (Fig. 2): $7.5 \mathrm{~cm}$ (T2 and T5), $10 \mathrm{~cm}$ (T1 and T4) and $12.5 \mathrm{~cm}$ (T3 and T6). 


\section{b. Data processing}

The temperature set point was reached more rapidly during heating than during cooling. As a result, a plateau was observed during heating, whereas the set point during cooling was only reached in the first cooling phase (Fig. 3). This dissymmetry arose from the fact that the heater and the cryostat that were used did not have the same capacities.

The raw data obtained from the test consisted of eight time series of temperature from each of the eight thermocouples. The measurements were synchronised and taken every minute. A running average algorithm was used to smooth the noisy raw data, except the temperature data for the absorber pipes. This running average algorithm involved averaging values over 20 time steps which represent $20 \mathrm{~min}$, centred on the value being smoothed.

The temperature of the heat-carrier fluid was not smoothed because doing so would have erased the sharp transition between heating and cooling and would have induced error in the determination of the phase shift. Indeed, the phase shift was estimated as the time required by the sharp change in the heating-cooling curve to propagate from the source to the monitoring points (Fig. 3). The temperature of the heat source (i.e. the absorber pipes) was approximated as the mean of the temperatures at the inlets and outlets of the absorber pipes.

An analysis of the spectral density of energy obtained from a fast Fourier transform of the time series was conducted to verify and retrieve the pulsation of the source and to ensure that this pulsation was not affected while propagating through the silt (Fig. 4). The peak energy density was found to occur at a frequency of $10^{-4.952} \mathrm{~Hz}$ for each thermocouple, which corresponds to a period of 1.04 days. Therefore, the applied period was $24 \mathrm{~h}$, as expected.

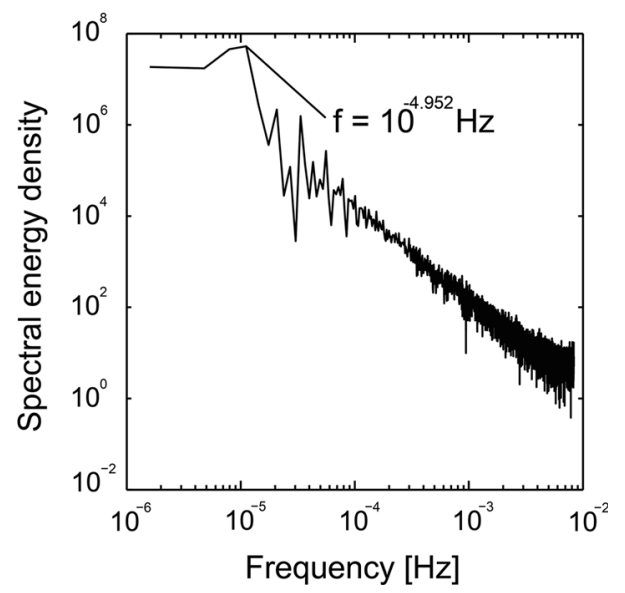

Fig. 4 Spectral density of energy of thermocouple T1
The amplitude attenuation was estimated for the last heating phase as the ratio between the amplitude of the response and the amplitude of the source (Fig. 3). The amplitude attenuation was estimated in this manner for each of the six thermocouples installed in the tank.

Two types of interference analysis were carried out. The first type is based on the comparisons between the source and the thermocouples and is called source-thermocouple interference analysis. The second type is based on the comparisons between the thermocouples and is called thermocouple-thermocouple interference analysis.

The time lag between the thermocouples in the thermocouple-thermocouple interference analysis was estimated using cross correlation by finding the maximum of the normalised cross covariance function based on the two thermocouple signals. However, determination of the time lag was more challenging for source-thermocouple interference analyses. Indeed, the presence of the plateau during the heating phase in the source signal would have overestimated the lag because cross correlation maximises the common area between the two curves instead of aligning the sharp transitions from heating to cooling which physically corresponds to the maximum temperature observed at the thermocouples (Fig. 3). Therefore, in the sourcethermocouple interference analyses, the time lag was estimated manually from the smoothed curves for the thermocouples, and a large margin of error, \pm 10 time steps (i.e. $\pm 10 \mathrm{~min})$, was assumed for the estimates. The phase shift was estimated as the lag between the last transition from heating to cooling at the source and the last positive peak observed at the thermocouple (Fig. 3).

\section{Analyses}

The analyses are divided into source-thermocouple interference analyses and thermocouple-thermocouple interference analyses.

a. Source-thermocouple interference analyses

As stated in Sect. 3.b, source-thermocouple interference analysis involves comparing the signal from the source with the responses measured by the different thermocouples.

Observed phase shifts and attenuations for the different thermocouples are presented in Table 1. Curves of phase shift and attenuation, as illustrated in Fig. 4, were produced for a source radius equal to $1 \mathrm{~cm}$-the diameter of the heat exchanger borehole-and values of $N\left(=r / r_{i}\right)$ for each thermocouple. In total, three sets of curves were used (one set per distance to the source for three different distances to the source; see Sect. 3.a). Four values of thermal diffusivity were derived per thermocouple: one from the amplitude attenuation $\left(D_{\delta}\right)$ and three from the phase shift: one from 
Table 1 Phase shift and attenuation from interference analyses between the source and the thermocouples

\begin{tabular}{lll}
\hline Sensor & $\varphi_{\mathrm{TT}}($ cycles $)$ & $\delta_{\mathrm{TT}}$ \\
\hline 1 & -0.1745 & 0.3205 \\
2 & -0.1309 & 0.3694 \\
3 & -0.3185 & 0.2626 \\
4 & -0.2269 & 0.3006 \\
5 & -0.1309 & 0.3656 \\
6 & -0.3185 & 0.2752 \\
\hline
\end{tabular}

the measured phase shift value $\left(D_{\varphi}\right)$ and two from the phase shift bounded by the error $\left(D_{\varphi-10}\right.$ and $\left.D_{\varphi+10}\right)$. These values were estimated only for assumed conditions of no heat flux at infinity, using Eq. (12), and no heat flux at the tank radius, using Eq. (11). The Dirichlet-type condition did not permit retrieval of any value of thermal diffusivity because the observed attenuations were greater than those allowed by Eqs. (13) or (10). This was attributed to the plateaux that were observed when heating, during which energy is injected while the source temperature (i.e. the mean between inlet and outlet temperatures) remains constant. The temperature increases that are thus observed in the silt are higher than those that would be observed if the source signal was really sinusoidal.

The results based on the lag between the source and the thermocouples with no heat flux at infinity yield a mean thermal diffusivity of $2.08 \times 10^{-7} \mathrm{~m}^{2} / \mathrm{s}$, bounded by $1.27 \times 10^{-7}$ and $4.26 \times 10^{-7} \mathrm{~m}^{2} / \mathrm{s}$ (Fig. 5a). The results based on the attenuation are likely to overestimate the retrieved thermal diffusivity because of the source signal shape, as detailed previously, and in this case yielded values one order of magnitude higher, with a mean value of $1.97 \times 10^{-6} \mathrm{~m}^{2} / \mathrm{s}$ (Fig. 5a). The results using the condition of no heat flux at the tank wall yielded mean estimates of $7.62 \times 10^{-7}$ and $5.94 \times 10^{-7} \mathrm{~m}^{2} / \mathrm{s}$, based on the phase shift and attenuation, respectively (Fig. 5b).

b. Thermocouple-thermocouple interference analyses

Thermocouple-thermocouple interference analyses were carried out by comparing the thermocouples with each other. Both attenuation and phase shift were analysed for the different pairs of thermocouples used. For a given pair, one thermocouple has to be identified as the source and one has to be identified as the monitoring point. For each pair of thermocouples, the thermocouple closer to the heat source was taken to be the new source, producing a source radius equal to the distance between this thermocouple and the tank axis. The second thermocouple (farther from the heat source) was then taken as the monitoring point. Only boundary conditions of the Neumann type were considered, for the reason discussed in the previous section.

Observed phase shift and attenuation between the thermocouple are presented in Tables 2 and 3, respectively, where the first column and raw represent the thermocouple number as shown in Fig. $2 b$.

The phase shift can be positive or negative, as the values in Table 2 represent the lag between the thermocouple reported in the first column and the thermocouple reported in the first raw. Obviously, thermocouples have no delay with respect to themselves, which explains the diagonals of zeroes in Table 2.

Amplitude attenuation (Table 3) can be $>1$. For example, if the signal at thermocouple 1 has an amplitude equal to 0.8672 times the amplitude at thermocouple 2 , then thermocouple 2 has an amplitude equal to $1 / 0.8672=1.1531$ times the amplitude at thermocouple 1. Obviously, comparing thermocouples to themselves yields no attenuation, so these ratios are equal to 1 .

Nevertheless, interference analyses were carried out considering only positive phase shifts and attenuations lower than 1 , which is consistent with selecting the thermocouple closer to the tank axis as the source and the thermocouple farther from the tank axis as the monitoring point.

Because cross correlation was used to estimate the phase shift, no margin of error was associated with the phase shift values, in contrast to the assumption of a margin of error for the manual time lag estimates described for the sourcethermocouple interference analyses.

Even if little attenuation is observed (Table 3) between thermocouples that are at the same distance from the tank
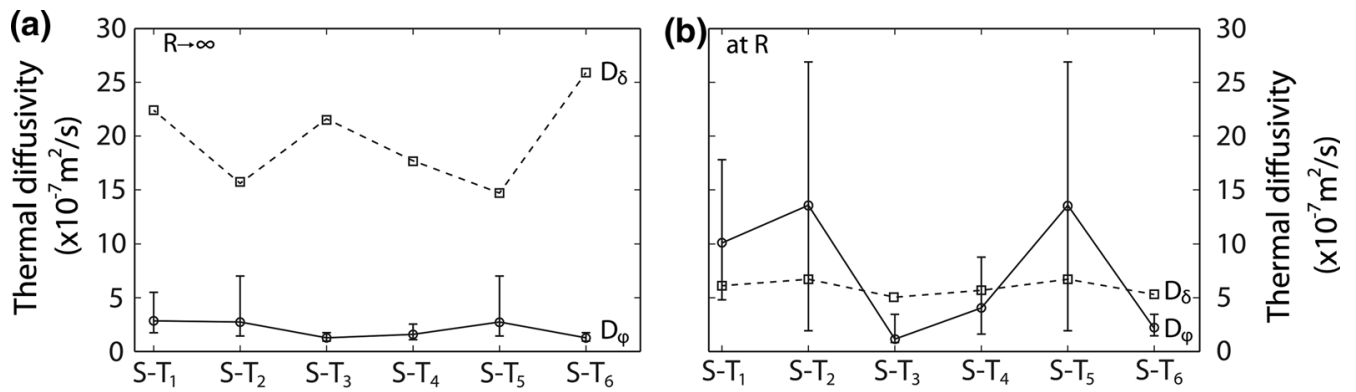

Fig. 5 Thermal diffusivity based on phase shift $\left(D_{\varphi}\right)$ and attenuation $\left(D_{\delta}\right)$ with the far-field condition of a no heat flux at infinity and $\mathbf{b}$ not heat flux at the tank wall 
Table 2 Phase shift between the different thermocouples

\begin{tabular}{llllllc}
\hline & 1 & 2 & 3 & 4 & 5 & 6 \\
\hline 1 & 0 & 0.092 & -0.079 & 0 & 0.092 & -0.061 \\
2 & -0.092 & 0 & -0.249 & -0.127 & 0 & -0.227 \\
3 & 0.079 & 0.249 & 0 & 0.048 & 0.244 & 0 \\
4 & 0 & 0.127 & -0.048 & 0 & 0.127 & -0.031 \\
5 & -0.092 & 0 & -0.244 & -0.127 & 0 & -0.227 \\
6 & 0.061 & 0.227 & 0 & 0.031 & 0.227 & 0 \\
\hline
\end{tabular}

The phase shift is given as the lag in number of cycles between the thermocouple of the first column and the thermocouple in the first row. A positive lag represents a delay

axis (the pairs T1-T4, T2-T5 and T3-T6), this was ignored and the attenuation was set to 1 for the rest of the analyses. This can come from the location of the thermocouples, either on one side of the other of the absorber pipes. The thermocouples on the hotter side (T1, T2 and T3), where the heat-carrier fluid goes downward, therefore, experience slightly greater temperature increase than the ones places on the colder side (T4, T5 and T6) where the fluid goes upward [24] (Fig. 2b).

Figure 6 gathers the results of the thermocouple-thermocouple interference analyses for the conditions of no heat flux at infinity $(R \rightarrow \infty)$ and at the tank wall $(R)$. The results are presented as boxplots. The dashed line in the box represents the median of the value set, the box edges are the 25 th and 75 th percentiles and the whiskers extend to extreme data not considered as outliers. The individual points are outliers. Despite these are plotted as outliers in the boxplots, they were included in the estimation of the mean values presented in Table 4. Individual values of estimated diffusivities are given in "Appendix" (Tables 5, $6,7,8)$.

The estimates based on the phase shift yield mean values of the thermal diffusivity of $1.24 \times 10^{-7} \mathrm{~m}^{2} / \mathrm{s}$ for the condition of no heat flux at infinity and $1.45 \times 10^{-7} \mathrm{~m}^{2} / \mathrm{s}$ for the condition of no heat flux at the tank wall.

The estimates based on attenuation yield mean values of the thermal diffusivity of $1.18 \times 10^{-5} \mathrm{~m}^{2} / \mathrm{s}$ for the condition of no heat flux at infinity and $2.39 \times 10^{-7} \mathrm{~m}^{2} / \mathrm{s}$ for the condition of no heat flux at the tank wall.

\section{Discussion}

Estimates based on the attenuation of the source signal across the soil mass are more scattered because of the deviation of the source signal from a real sine wave (Table 4). The results therefore show that the phase shift analysis does not really require a perfect shape of the source signal, though the shape of the source signal does has a significant impact on the attenuation analysis.

Based on the phase shift only, the estimated thermal diffusivity of the silt was found to be, on average, $3.1 \times 10^{-7} \mathrm{~m}^{2} / \mathrm{s}$, whereas the estimated value based on the attenuation was higher: $3.65 \times 10^{-6} \mathrm{~m}^{2} / \mathrm{s}$.

Al Nakshabandi and Kohnke [1] estimated the thermal diffusivity of a silt using Eq. (3) by measuring the bulk density and thermal conductivity of samples and assuming an average specific heat value of $0.199 \mathrm{cal} / \mathrm{g} /{ }^{\circ} \mathrm{C}$ (i.e. $833 \mathrm{~J} / \mathrm{kg} / \mathrm{K}$ ) which are "averages of data obtained in the literature". The thermal conductivity was measured using a thermal probe at different soil moisture contents. The estimates of thermal diffusivity obtained by these researchers range from $1.5 \times 10^{-7}$ to $7.0 \times 10^{-7} \mathrm{~m}^{2} / \mathrm{s}$ for moisture contents going from 0 to $35 \%$ (i.e. dry to saturated). Wolfe and Thieme [23] measured the thermal conductivity and specific heat capacity of samples of silt at various temperatures in nearly saturated conditions (i.e. moisture content of 17-22\%). The thermal conductivity was measured using a cylindrical configuration (see Farouki [7]). Within the temperature range considered in the present study, Wolfe and Thieme estimated a thermal conductivity of approximately $0.5 \mathrm{BTU} / \mathrm{h} / \mathrm{ft} /{ }^{\circ} \mathrm{F}(0.865 \mathrm{~W} /$ $\mathrm{m} / \mathrm{K}$ ) and a specific heat of approximately $0.3-0.4 \mathrm{BTU} / \mathrm{lb} /$ ${ }^{\circ} \mathrm{F}(1,256-1,674 \mathrm{~J} / \mathrm{kg} / \mathrm{K})$. The combination of these measurements with a soil grain density of $2,700 \mathrm{~kg} / \mathrm{m}^{3}$ leads to values of thermal diffusivity of approximately $3.0 \times 10^{-7} \mathrm{~m}^{2} / \mathrm{s}$, using Eq. (3). Therefore, the estimates obtained in this study on the basis of the phase shift seem to

Table 3 Attenuations between the thermocouples

\begin{tabular}{lllllll}
\hline & 1 & 2 & 3 & 4 & 5 & 6 \\
\hline 1 & 1 & 0.8672 & 1.2177 & 1.0642 & 1.2272 & 1.1615 \\
2 & 1.1531 & 1 & 1.4042 & 0.8739 & 1.0101 & 0.7194 \\
3 & 0.8212 & 0.7121 & 1 & 1 & 0.8231 & 1 \\
4 & 0.9397 & 0.8149 & 1.1443 & 1.2149 & 1.9538 \\
5 & 1.1416 & 0.99 & 1.3901 & 0.9163 & 0.7542 \\
6 & 0.861 & 0.7466 & 1.0485 & 1.3259 \\
\hline
\end{tabular}

Values can be $>1$ because thermocouples in the first column are compared with thermocouples in the first row 


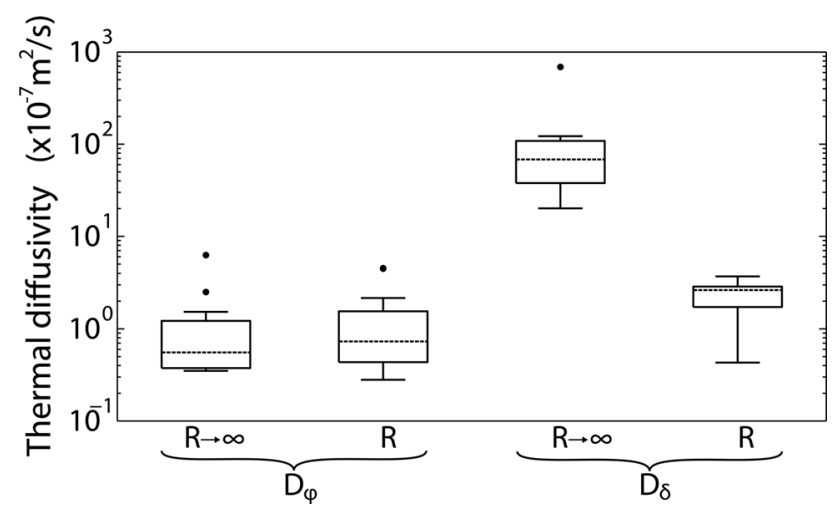

Fig. 6 Results of the interference analyses between thermocouples based on phase shift $\left(D_{\varphi}\right)$ and attenuation $\left(D_{\delta}\right)$ with no heat flux at infinity $(R \rightarrow \infty)$ and at the thank wall $(R)$

be more consistent with the values reported in the literature, while the estimates obtained on the basis of attenuation are, as expected, greater.

However, deploying this test procedure at full-scale would be challenging for different reasons. First, the accurate deployment of the temperature sensor at a given distance from the absorber pipes requires perfectly parallel drilling which is limited in long boreholes. Sensitivity of the method to the source-thermocouple distance was tested for the thermocouples $\mathrm{T} 2$ and $\mathrm{T} 5$ at a radius of $7.5 \mathrm{~cm}$. Thermal diffusivities were estimated using inversion curves as in Fig. 1 for distances of $6.5,7.5$ and $8.5 \mathrm{~cm}$, allowing an error of $1 \mathrm{~cm}$ corresponding to $13 \%$ and considering no flux at infinity. Results are (Fig. 7):

- Based on phase shift, thermal diffusivity is bounded between $1.75 \times 10^{-7}$ and $3.48 \times 10^{-7} \mathrm{~m}^{2} / \mathrm{s}$

- Based on attenuation, thermal diffusivity is bounded between $1.15 \times 10^{-6}$ and $5.78 \times 10^{-6} \mathrm{~m}^{2} / \mathrm{s}$. These results should be tempered as they were shown not to be consistent because of the source signal shape deviating from a sine wave.

Therefore, the technique is sensitive to the location of the monitoring points.

Second, deploying this technique at full-scale will be more time-consuming than with the scaled model and would involve the installation of temperature sensors in boreholes. Because TRTs do not require thermal cycles, they are not totally compatible with the tested method. However, the heat source is the same for a TRT and for a pumping test (i.e. a geothermal borehole). Therefore, the TRT could represent the first heating of a series of cycles that would be used for the pumping test. Typical time span of a TRT is 1 week [15] so that would represent a half cycle. Then, considering that three cycles are required to reach the forced periodic regime, a total time span of 6 weeks should be required to carry this test at full-scale, which is consequent, but consistent with the experience of Renner and

Table 4 Summary of the mean values of thermal diffusivity retrieved by the different analysis approaches

\begin{tabular}{lllll}
\hline & $\begin{array}{l}\text { Source-thermocouple } \\
\text { NHF }^{\mathrm{a}} \text { at } R \rightarrow \infty\end{array}$ & $\begin{array}{l}\text { Source-thermocouple } \\
\mathrm{NHF}^{\mathrm{a}} \text { at } R_{\text {wall }}\end{array}$ & $\begin{array}{l}\text { Thermocouple-thermocouple } \\
\text { NHF }^{\mathrm{a}} \text { at } R \rightarrow \infty\end{array}$ & $\begin{array}{l}\text { Thermocouple-thermocouple } \\
\text { NHF }^{\mathrm{a}} \text { at } R_{\text {wall }}\end{array}$ \\
\hline$D_{\varphi}\left(\mathrm{m}^{2} / \mathrm{s}\right)$ & $2.08 \times 10^{-7}$ & $7.62 \times 10^{-7}$ & $1.24 \times 10^{-7}$ & $1.45 \times 10^{-7}$ \\
$D_{\delta}\left(\mathrm{m}^{2} / \mathrm{s}\right)$ & $1.97 \times 10^{-6}$ & $5.94 \times 10^{-7}$ & $1.18 \times 10^{-5}$ & $2.39 \times 10^{-7}$ \\
\hline
\end{tabular}

${ }^{\mathrm{a}} \mathrm{NHF}$ no heat flux
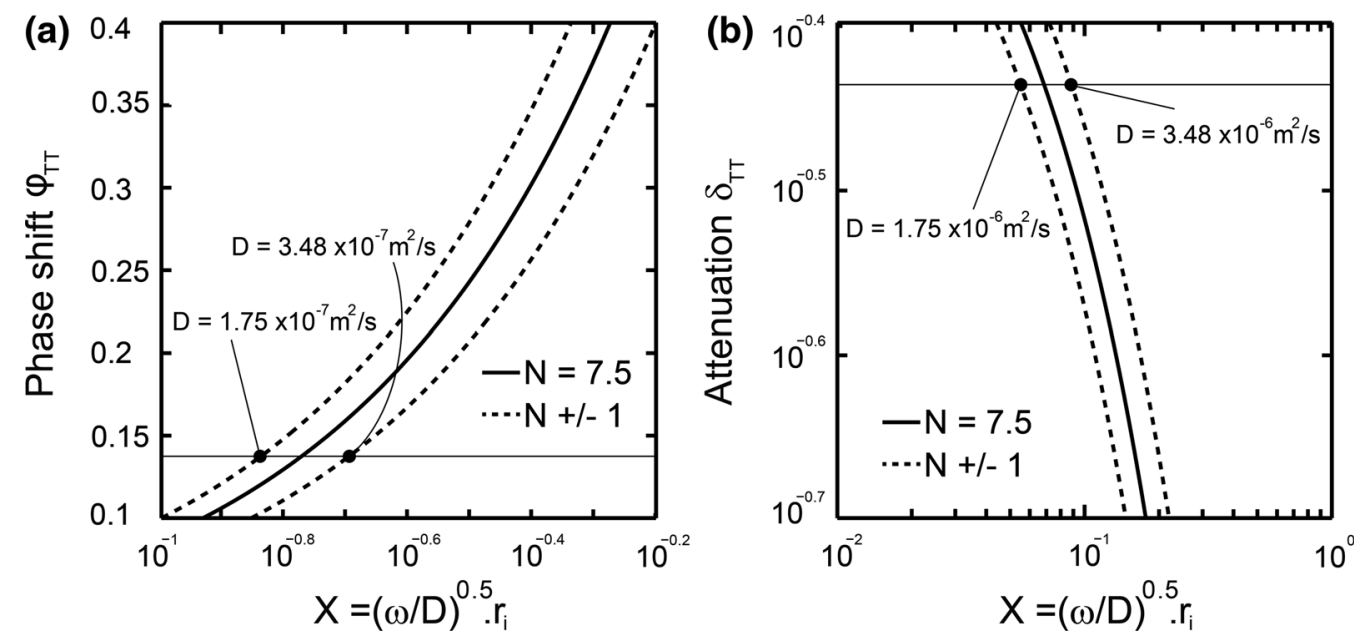

Fig. 7 Curves used for graphical inversion of Eq. (14) for the thermocouples T2 and T5 (solid lines) and curves with and error of $\pm 13 \%$ (dashed lines). Horizontal lines represent the observed phase shift and attenuation as reported in Table 1 
Messar [18]. Indeed, because hydraulic diffusivity is 6-7 orders of magnitudes higher than thermal diffusivity, much more time is required for the test.

A clear signal (i.e. with significant amplitude) at the monitoring points is required to obtain good estimates of phase shift and attenuation. The accuracy of commercial thermistors or thermocouples designed for underground installations is roughly $0.1{ }^{\circ} \mathrm{C}$ while amplitude of heat source temperature increase during a TRT is between 10 and $20{ }^{\circ} \mathrm{C}$. Therefore, the monitoring points should not be too far from the source to avoid significant attenuation, and in the same time, should not be too close to the source as the thermal impact of the filling material of the installation borehole would induce greater bias. Figure 8 was drawn to estimate the optimal source-sensor distance based on order of magnitudes. It represents the quadratic evolution of the characteristic pulse time according to the characteristic dimension (i.e. source-monitoring point distance). The curves are based on the dimensionless heat equation that yields:

$D_{\mathrm{T}} \sim \frac{L^{2}}{\tau} \Leftrightarrow \tau \sim \frac{L^{2}}{D_{\mathrm{T}}}$

where $L$ and $\tau$ are the characteristic length (i.e. radius) and time (i.e. heat source period) on which heat conduction occurs. Therefore, the two bounding curves presented in Fig. 8 are parabolas using $D_{\mathrm{T}}=10^{-7}$ and $D_{\mathrm{T}}=10^{-6} \mathrm{~m}^{2} / \mathrm{s}$.

A signal period of 1 day is shown to generate good signals at the monitoring points for the scale of the scaled model used in this study (i.e. source-sensor distance of approximately $10 \mathrm{~cm}$ ). Using a signal period of 1 week at the heat source for the full-scale application leads to a favourable source-sensor distance between 0.2 and $0.8 \mathrm{~m}$. However, the portion of borehole filling material is significant for low distances as the borehole diameters are generally between 0.1 and $0.2 \mathrm{~m}$ [17]. Therefore, a reasonable range of source-sensor distance is between 0.5 and $0.8 \mathrm{~m}$, wherein the portion of borehole filling material

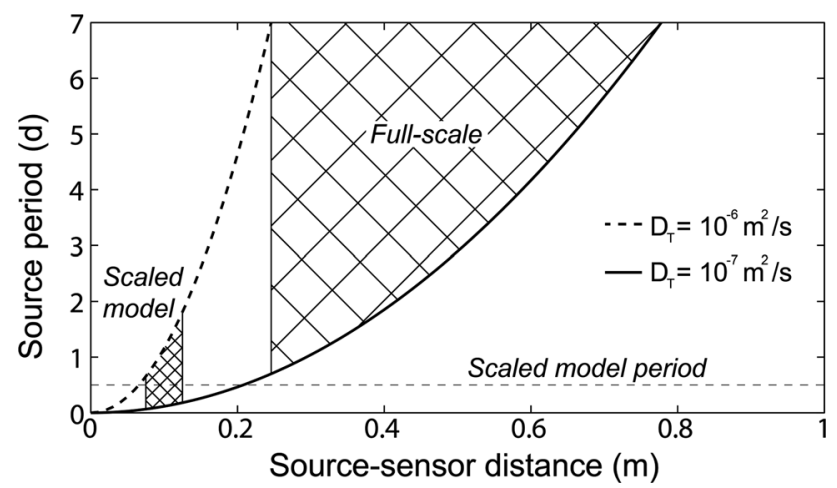

Fig. 8 Relationship between time and space scales for heat conduction in soils with thermal diffusivities between $10^{-7}$ and $10^{-6} \mathrm{~m}^{2} / \mathrm{s}$ varies between 10 and $20 \%$. The bias introduced by the thermal influence of the filling material could be accounted for as its thermal properties are known but no particular solution is provided herein.

The vertical inhomogeneity should also be assessed for the deployment at full-scale. Indeed, several temperature sensors should be installed along the monitoring borehole when different soil layers are encountered. When local stratigraphy is well documented, at least one temperature sensor should be placed in each of the identified soil layers. Furthermore, provided the relatively low cost of thermistors or thermocouples compared with the cost of a borehole should encourage installing at least 5-10 temperature sensors.

Despite some extra installation requirements and a longer testing time if deployed on a larger scale, this test would be of great value in the design of thermal foundations that reach depths of approximately $10-30 \mathrm{~m}$ and have narrow spacing between the heat exchanger structures. Indeed, seasonal operations could dramatically affect the sustainability of the system if the inertia of the ground was not taken into account properly in the design phase.

Finally, Renner and Messar [18] suggest that using different periods of input signal may also allow investigating the intrinsic period dependence of the soil thermal properties as both daily and yearly operations may have a significant impact on ground heat exchangers.

\section{Conclusions}

A method based on periodic pumping tests was evaluated using a scaled model of a geothermal borehole for the purpose of in situ estimation of the bulk thermal diffusivity of a soil. The potential of this method is demonstrated on the scaled boreholes. The thermal diffusivity estimates based on the phase shift exhibit good agreement between each other and with estimates found in the literature. Analyses based on the attenuation of the source signal should not be considered if the source signal deviates too much from a sine-like signal. The limitations of deploying the presented method at full-scale were discussed. A time period of 4-6 weeks should be required to reach a forced periodic regime over 2-3 heating/cooling cycles of 2 weeks each. The heat source-sensor distance is found to be an important parameter as the portion of filling material between the source and the temperature sensor may significantly influence the finally retrieved thermal diffusivity. Therefore, this portion should be kept as minimal as possible. Finally, the sensitivity of the method to the accurate localisation of the sensor was investigated based on the results of the scaled model. An error of $13 \%$ in the heat source-sensor distance leads to an error of $50 \%$ in the final estimates based on phase shift analyses. Furthermore, 
vertical stratigraphy of the ground must be accounted for by deploying several temperature sensors along the monitoring borehole.

In conclusion, the method potential was clearly evidenced on the scaled model but several limitations remain to be overcome for the full-scale deployment.

Acknowledgments The authors would like to thank EOS Holding for its financial support through the project GRETEL II: Geotechnical Reliability of Thermo-Pile Energy Storage in Soils.

\section{Appendix}

The individual estimates represented in Fig. 6 are detailed in this appendix. The results were split into four different tables to clarify their contents. Table 5 presents estimates based on the phase shift and the boundary condition of no heat flux at infinity. Table 6 presents estimates based on the phase shift and the boundary condition of no heat flux at the tank wall. Table 7 presents the estimates based on the attenuation and the boundary condition of no heat flux

Table 5 Values of retrieved thermal diffusivity $D_{\varphi}$ (in $10^{-7} \mathrm{~m}^{2} / \mathrm{s}$ ) based on phase shift and no heat flux at infinity

\begin{tabular}{llllr}
\hline & 1 & 3 & 4 & 5 \\
\hline 1 & 0.67 & 0.91 & & 0.67 \\
2 & & 0.37 & 0.35 & 0.53 \\
3 & & & 2.51 & 0.38 \\
4 & & & 0.35 & \\
5 & & & & 0.45 \\
6 & & & & \\
\hline
\end{tabular}

Table 6 Values of retrieved thermal diffusivity $D_{\varphi}$ (in $10^{-7} \mathrm{~m}^{2} / \mathrm{s}$ ) based on phase shift and no heat flux at $R$

\begin{tabular}{llllll}
\hline & 1 & 2 & 3 & 4 & 5 \\
\hline 1 & 1.53 & 1.57 & & 1.53 & \\
2 & & 0.53 & 0.34 & 0.73 \\
3 & & & 0.28 & 0.57 \\
4 & & & 0.34 & \\
5 & & & & 0.731 \\
6 & & & & \\
\hline
\end{tabular}

Table 7 Values of retrieved thermal diffusivity $D_{\delta}$ (in $10^{-7} \mathrm{~m}^{2} / \mathrm{s}$ ) based on attenuation and no heat flux at infinity

\begin{tabular}{|c|c|c|c|c|c|c|}
\hline & 1 & 2 & 3 & 4 & 5 & 6 \\
\hline 1 & & 122 & 20.1 & & 122 & 54.8 \\
\hline 2 & & & 43.9 & 26.2 & & 81.6 \\
\hline 3 & & & & 83.4 & 49.6 & \\
\hline 4 & & & & & 31.7 & 688 \\
\hline 5 & & & & & & 94.9 \\
\hline 6 & & & & & & \\
\hline
\end{tabular}

Table 8 Values of retrieved thermal diffusivity $D_{\delta}$ (in $10^{-7} \mathrm{~m}^{2} / \mathrm{s}$ ) based on attenuation and no heat flux at $R$

\begin{tabular}{|c|c|c|c|c|c|c|}
\hline & 1 & 2 & 3 & 4 & 5 & 6 \\
\hline 1 & & 3.55 & 1.41 & & 3.71 & 1.67 \\
\hline 2 & & & 2.50 & 2.82 & & 2.77 \\
\hline 3 & & & & 1.78 & 0.43 & \\
\hline 4 & & & & & 2.91 & 2.27 \\
\hline 5 & & & & & & 2.84 \\
\hline 6 & & & & & & \\
\hline
\end{tabular}


condition at infinity. Table 8 presents the estimates based on the attenuation and the boundary condition of no heat flux at the tank wall. Obviously, no estimation can be achieved by comparing a thermocouple to itself or to the other thermocouple at the same distance from the tank axis (the pairs T1-T4, T2-T5 and T3-T6).

\section{References}

1. Al Nakshabandi G, Kohnke H (1965) Thermal conductivity and diffusivity of soils as related to moisture tension and other physical properties. Agric Meteorol 2:271-279

2. Amercian Society for Testing and Materials (1963) Thermal conductivity of materials by means of guarded hot plate. ASTM Specification C177-63

3. Axelsson G (2010) Sustainable geothermal utilization-case histories; definitions; research issues and modelling. Geothermics 39(4):283-291

4. Brandl H (2006) Energy foundations and other thermo-active ground structures. Géotechnique 56(2):81-122

5. Bristow KL, Campbell GS, Calissendorff K (1993) Test of a heatpulse probe for measuring changes in soil water content. Soil Sci Soc Am J 57(4):930-934

6. Eskilson P (1987) Thermal analysis of heat extraction boreholes. Ph.D. Thesis, University of Lund, Department of Mathematical Physics, Lund, Sweden

7. Farouki OT (1986) Thermal properties of soils. Trans Tech Pub, Rockport

8. Forbes JD (1849) Account of some experiments on the temperature of the Earth at different depths, and in different soils, near Edinburgh. Trans R Soc Edinb 10:189-236

9. Garber D, Choudhary R, Soga K (2013) Risk based lifetime costs assessment of a ground source heat pump (GSHP) system design: methodology and case study. Build Environ 60:66-80
10. Hermansson $\AA$, Charlier R, Collin F, Erlingsson S, Laloui L, Sršen MS (2009) Heat transfer in soils. In: Andrew D (ed) Water in road structures. Springer, Netherlands, pp 69-79

11. Hinkel KM (1997) Estimating seasonal values of thermal diffusivity in thawed and frozen soils using temperature time series. Cold Reg Sci Technol 26(1):1-15

12. Hoekstra P, Delaney A, Atkins R (1973) Measuring the thermal properties of cylindrical specimens by the use of sinusoidal temperature waves. CRREL Technical Report 244, AD 770425

13. Kersten MB (1949) Laboratory research for the determination of the thermal properties of soils. ACFEL Technical Report 23, AD 712516

14. Laloui L, Di Donna A (eds) (2013) Energy geostructures innovation in underground engineering. Wiley-ISTE, Hoboken

15. Mattsson N, Steinmann G, Laloui L (2008) Advanced compact device for the in situ determination of geothermal characteristics of soils. Energy Build 40(7):1344-1352

16. Mochlinski K (1964) Some industrial measurements of thermal properties of soil. In: International study group on soils. Cambridge, England, July 12-26, pp 168-178

17. Pahud D, Matthey B (2001) Comparison of the thermal performance of double U-pipe borehole heat exchangers measured in situ. Energy Build 33(5):503-507

18. Renner J, Messar M (2006) Periodic pumping tests. Geophys J Int 167:479-493

19. Sanner B, Karytsas C, Mendrinos D, Rybach L (2003) Current status of ground source heat pumps and underground thermal energy storage in Europe. Geothermics 32(4-6):579-588

20. Scott RF (1964) Heat exchange at the ground surface. Cold Reg Sci Eng Monogr II-D1, AD 697136

21. Shannon WL, Wells WA (1947) Tests for thermal diffusivity of granular materials. In: Proceedings of the American society for testing and materials, vol 47, pp 1044-1055

22. Williams GP, Gold LW (1976) Ground temperatures. Can Build Dig 180

23. Wolfe LM, Thieme JO (1964) Physical and thermal properties of frozen soils and ice. J Soc Petrol Eng 4(1):67-72

24. Zeng H, Diao N, Fang Z (2003) Heat transfer analysis of boreholes in vertical ground heat exchangers. Int J Heat Mass Transf 46(23):4467-4481 